\title{
EMBEDDING THEOREMS AND GENERALIZED DISCRETE ORDERED ABELIAN GROUPS
}

\author{
BY
}

\author{
PAUL HILL AND JOE L. MOTT
}

\begin{abstract}
Let $G$ be a totally ordered commutative group. For each nonzero element $g \in G$, let $L(g)$ denote the largest convex subgroup of $G$ not containing $g$. Denote by $U(g)$ the smallest convex subgroup of $G$ that contains $g$. The group $G$ is said to be generalized discrete if $U(g) / L(g)$ is order isomorphic to the additive group of integers for all $g \neq 0$ in $G$. This paper is principally concerned with the structure of generalized discrete groups. In particular, the problem of embedding a generalized discrete group in the lexicographic product of its components, $U(g) / L(g)$, is studied. We prove that such an embedding is not always possible (contrary to statements in the literature). However, we do establish the validity of this embedding when $G$ is countable. In case $F$ is $o$-separable as well as countable, the structure of $G$ is completely determined.
\end{abstract}

1. Notation and terminology. All groups considered here in are abelian. Let $G$ be a totally ordered abelian group. The set $\mathcal{S}$ of all convex subgroups of $G$ is linearly ordered by containment. The order rank of $G$ is by definition the order type of the set $\mathcal{S} \backslash\{G\}$. If $D$ and $C$ are convex subgroups of $G$ such that $D \subset C$ and if there is no convex subgroup between $D$ and $C$, then we say that $C$ covers $D$ and that $C$ is a principal convex subgroup of $G$. We refer to the extension $D \subset C$, when $C$ covers $D$, as a jump. Let $P$ be a set indexing the collection $\delta_{0}$ of principal convex subgroups $C_{\pi}$ of $G$, where $\pi \leq \rho$ if and only if $C_{\pi} \supseteq C_{\rho}$. If $C_{\pi}$ covers $D_{\pi}$, let $R_{\pi}=C_{\pi} / D_{\pi}$; the skeleton of $G$ is the system $\left[P, R_{\pi}(\pi \in P)\right]$, and we call $R_{\pi}$ a component of $G$. If $0 \neq t \in G$, let $U(x)$ denote the intersection of all convex subgroups of $G$ containing $x$, and let $L(x)$ denote the union of all convex subgroups not containing $x$. Clearly $L(x) \subset U(x)$ is a jump, and all jumps can be obtained in this manner. Two elements $a$ and $b$ of an ordered abelian group $G$ are said to be archimedean equivalent if there exist positive integers $m$ and $n$ such that $|a|<m|b|$ and $|b|<n|a|$. Clearly $a$ and $b$ are archimedean equivalent if and only if $U(a)=U(b)$ and $/$ or $L(a)=$ $L(b)$.

Suppose that $G$ is abelian, $H \subseteq G$, and that $H$ and $G / H$ are totally ordered. The positive elements of $H$ and all the elements of $G$ belonging to positive

Received by the editors June 10, 1971.

AMS (MOS) subject classifications (1970). Primary 06A60.

Key words and phrases. Totally ordered group, discrete group, generalized discrete, Hahn's embedding theorem, regular group. 
cosets in $G / H$ define a total order on $G$ so that $H$ is a convex subgroup of $G$. Moreover, the skeleton of $G$ is completely determined in the obvious way by the skeletons of $H$ and $G / H[17]$.

When we say that an ordered group $G$ algebraically has a certain property, we mean that the group has the property as an unordered group. For example, the statement that $G$ is algebraically free means simply that $G$ is a direct sum of infinite cyclic groups; it says nothing about the way that $G$ is ordered.

Finally, the symbols $\mathrm{Il}$ and $\Sigma$ denote the direct product and direct sum, respectively. For other definitions the reader should consult Fuch's book [10].

2. The embedding problem for generalized discrete groups. One would like to determine conditions under which an ordered group $G$ can be embedded in the lexicographic product of its components (or small extensions of the components). The best known result along this line is Hahn's embedding theorem, proved by Hahn in 1907 [14]. Since then several generalizations ([3], [5], [6], [7], [13], [15]) have appeared.

Following Ribenboim [20], we define a regular group as a totally ordered abelian group $G$ such that, for each $\pi \in P, R_{\pi}$ is a free $A$-module for some ring $A$ such that $Z \subseteq A \subseteq Q$. In [20], Ribenboim erroneously asserts that any regular group admits an order embedding into the lexicographic product of its components. The error is retained in [21]. We give in Theorem 3.1 the first of two essentially different counterexamples. Although we shall show in $\$ 3$ that Ribenboim's theorem is false in the generality for which he states it, the following more restricted version seems plausible.

Define an ordered group $G$ to be a generalized discrete group if each component in the skeleton of $G$ is order isomorphic to the integers. We suggest that it is reasonable to conjecture that every generalized discrete group can be embedded in the lexicographic product of its components-that is, in the lexicographic product of copies of $Z$. Credibility is lent to such a conjecture by the suggestion that the projectivity of the components of a generalized discrete group ought to produce the desired result in a manner dual to the way that injectivity is used in the proof of Hahn's embedding theorem. However, the proposed conjecture again is false (Theorem 3.4), but we establish in $\$ 5$ its validity in case $G$ is countable.

A remark on the choice of our terminology "generalized discrete group" (abbreviated "g. d. group") is perhaps in order. We introduce this terminology cognizant of the fact that by a discrete totally ordered group $G$ some authors (see [18], [22], [23], [24]) mean that $G$ has a least positive element. Thus a discrete group, in this sense, need not be a generalized discrete group, according to our definition. However, our point of view is the following. We think of 
$Z$, the ordered group of integers, as being the standard model for a discrete group. A natural generalization is a group whose components are all isomorphic to $Z$. Obviously, a discrete group, as defined above, may fail to have this property. Indeed, precisely all that is required for a totally ordered group to be discrete (that is, have a least positive element) is for the group to be an extension of $Z$ by any totally ordered group. And, of course, there are such groups, even among the split extensions $X \oplus Z$, whose global structure is only remotely similar to that of $Z$; whereas our g. d. groups, particularly the countable ones, have a much closer relationship with $Z$.

\section{Counterexamples to Ribenboim's theorem.}

Theorem 3.1. There is a regular group $G$ with components $Z$ and $Q$ which cannot be embedded in $Q \oplus Z$.

Proof. Any extension of $Z$ by $Q$ can be ordered in such a way that the skeleton of $G$ is $R_{1}=Q$ and $R_{2}=Z$; in particular, $G$ can be ordered so that it is a regular group. Thus the proof of the theorem is contained in the following proposition. Most, if not all, of the proposition is well known; however, we include the proof for completeness. The proposition deals with unordered groups.

Proposition 3.2. Ext $(Q, Z)$ is the direct sum of $c$ copies of $Q$. Any subgroup of $Q \oplus Z$ of torsion free rank 2 is decomposable into a direct sum of the form $A \oplus Z$, where $A$ bas torsion free rank 1 . No nonsplit extension of $Z$ by $Q$ can be embedded in $Q \oplus Z$.

Proof. The exact sequence $0 \rightarrow Z \rightarrow Q \rightarrow Q / Z \rightarrow 0$ gives rise to the exact sequence

$$
\begin{aligned}
0 & \rightarrow \operatorname{Hom}(Q, Z) \rightarrow \operatorname{Hom}(Q, Q) \rightarrow \operatorname{Hom}(Q, Q / Z) \\
& \rightarrow \operatorname{Ext}(Q, Z) \rightarrow \operatorname{Ext}(Q, Q) \rightarrow \operatorname{Ext}(Q, Q / Z) \rightarrow 0 .
\end{aligned}
$$

However, $\operatorname{Hom}(Q, Z)=0$ since $Q$ is divisible and $Z$ is reduced, and $\operatorname{Ext}(Q, Q)$ $=0$ since $Q$ is divisible (hence injective). Thus we have the exact sequence

$$
0 \rightarrow \operatorname{Hom}(Q, Q) \rightarrow \operatorname{Hom}(Q, Q / Z) \rightarrow \operatorname{Ext}(Q, Z) \rightarrow 0 .
$$

Clearly, $\operatorname{Hom}(Q, Q)$ is isomorphic to $Q$. Hence to show that $\operatorname{Ext}(Q, Z)$ is the direct sum of $c$ copies of $Q$, it suffices to show that $\operatorname{Hom}(Q, Q / Z)$ is itself a direct sum of $c$ copies of $Q$. Observe that $\operatorname{Hom}(Q, Q / Z)$ is a vector space over $Q$, so it is enough to show that $\operatorname{Hom}(Q, Q / Z)$ has cardinality $c$. But any homomorphism of $Q / Z$ into $Q / Z$ gives rise to one from $Q$ into $Q / Z$, so the result will follow if $\operatorname{Hom}(Q / Z, Q / Z)$ is of cardinality $c$. Since $Q / Z=$ $\Sigma_{\text {primes }} Z\left(p^{\infty}\right)$, we need only observe that $\operatorname{Hom}\left(Z\left(p^{\infty}\right), Z\left(p^{\infty}\right)\right)$ has cardinality 
c. However, it is well known that $\operatorname{Hom}\left(Z\left(p^{\infty}\right), Z\left(p^{\infty}\right)\right)$ is the additive group of $p$-adic integers, a torsion free group having cardinality of the continuum.

Next, we assume that $G$ is a subgroup of $Q \oplus Z$ of torsion free rank two. Then $Q \cap G \neq G$ and $G / Q \cap G$ is isomorphic to a subgroup of $Q \oplus Z / Q \simeq Z$. Hence $G \simeq(Q \cap G) \oplus Z$.

Finally, suppose that $G$ is a nonsplit extension of $Z$ by $Q$; we have already shown that a continuum number of such extensions exist. Let $Z=\langle c\rangle$. Then we have the exact sequence $0 \rightarrow\langle c\rangle \rightarrow G \rightarrow Q \rightarrow 0$ which does not split. If $G$ can be embedded in $Q \oplus Z$, then we have shown that $G=A \oplus B$ where one of the groups $A$ or $B$ is infinite cyclic, and both $A$ and $B$ are nonzero. If $c$ is in $A$, then

$$
G /\langle c\rangle \simeq A /\langle c\rangle \oplus B \simeq Q
$$

and thus, since $Q$ is indecomposable, $A=\langle c\rangle$ and $G$ would be a split extension of $Z=\langle c\rangle$ by $Q$. This implies that $c=a+b$, where $a$ and $b$ are nonzero elements of $A$ and $B$, respectively. Thus $Q \simeq G /\langle c\rangle \stackrel{b}{\longrightarrow} A /\langle a\rangle \oplus B /\langle b\rangle \rightarrow 0$ is exact where $b$ has nontrivial kernel. It follows that $A /\langle a\rangle$ and $B /\langle b\rangle$ are torsion and divisible. Since one of $A$ and $B$, say $B$, is cyclic, it follows that $B=$ $\langle b\rangle$. Thus under the natural homomorphism from $G$ to $G /\langle b\rangle \simeq A$ the element $c$ is mapped to $a$. Hence $Q /\langle\bar{b}\rangle \simeq G /\langle c, b\rangle \simeq A /\langle a\rangle$, which implies that $A \simeq Q$ [11]. However, in this case we may assume that $c \in B$, and the proof of the theorem is finished.

Corollary 3.3. Not every regular group can be embedded in the lexicographic product of its components.

The above is perhaps the simplest counterexample of Ribenboim's theorem, but the next example is much more interesting. It also defeats our proposed conjecture for generalized discrete groups.

Theorem 3.4. There exists a generalized discrete group $G$ that cannot be embedded in a product of integers, even as an unordered group.

Proof. One remark is pertinent before we start the actual proof of the theorem. If $H$ and $K$ are g.d. groups, and if $G \in \operatorname{Ext}(H, K)$, then as we mentioned, $G$ can be ordered so that its skeleton is completely determined by the skeletons of $H$ and $K$, respectively. Thus if $H$ and $K$ are g.d. groups and if $G \in \operatorname{Ext}(H, K)$, then $G$ admits a total order such that it, too, is a g.d. group. Our plan is to show that there is a group $G$ in $\operatorname{Ext}(P, Z)$ which cannot be embedded in a product of integers, where $P$ is the cartesian product of countably many copies of the integers.

The structure of $\operatorname{Ext}(P, Z)$ is completely determined by Nunke in [19]. 
Here, however, it is enough to know that $\operatorname{Ext}(P, Z)$ has elements of infinite order; the proof of this fact follows easily from the fact that $\operatorname{Ext}(Q, Z)=\Sigma_{c} Q$, which is contained in Proposition 3.2. For there exists the exact sequence

$$
0 \rightarrow S \rightarrow P_{0} \rightarrow \sum_{c} Q \rightarrow 0,
$$

where $S=\Sigma_{\mathcal{N}_{0}} Z$ and $P_{0} \subseteq P$, and hence there exists an exact sequence

$$
\operatorname{Hom}(S, Z) \rightarrow \operatorname{Ext}\left(\sum_{c} Q, Z\right) \rightarrow \operatorname{Ext}\left(P_{0}, Z\right) \rightarrow 0
$$

$P_{0}$ is simply the closure of $S=\Sigma_{\boldsymbol{N}_{0}} Z$ in $P=\Pi_{\boldsymbol{\kappa}_{0}} Z$ in the $Z$-adic topology. We observe that $\operatorname{Ext}\left(\Sigma_{c} Q, Z\right)=\Pi_{c}(\operatorname{Ext}(Q, Z))$ is torsion free and has cardinality $2^{c}$, whereas $\operatorname{Hom}(S, Z)$ has cardinality $c$. Thus $\operatorname{Ext}\left(P_{0}, Z\right)$ must itself contain $2^{c}$ copies of $Q$, and it follows that $\operatorname{Ext}(P, Z)$ has elements of infinite order in view of the epimorphism: $\operatorname{Ext}(P, Z) \rightarrow \operatorname{Ext}\left(P_{0}, Z\right)$.

Now let

$$
0 \rightarrow\langle c\rangle \rightarrow G \rightarrow G /\langle c\rangle \rightarrow 0
$$

represent an element of infinite order in $\operatorname{Ext}(P, Z)$. From the above remarks, $G$ admits a g. d. structure. We claim that $G$ cannot be embedded in a product of integers. Assume that $G \subseteq \Pi_{\lambda \in \Lambda}\left\langle x_{\lambda}\right\rangle$. Since $\Pi_{\lambda \in \boldsymbol{\Lambda}}\left\langle x_{\lambda}\right\rangle$ is separable [11, p. 168], we can write

$$
\prod_{\lambda \in \Lambda}\left\langle x_{\lambda}\right\rangle=\prod_{\mu \in M}\left\langle y_{\mu}\right\rangle \oplus\langle b\rangle
$$

where $n b=c$ for some positive integer $n$. This means that the sequence

$$
0 \rightarrow\langle c\rangle \rightarrow\langle n G, c\rangle \rightarrow\langle n G, c\rangle /\langle c\rangle \rightarrow 0
$$

must split, for $\langle n G, c\rangle=\left(\langle n G, c\rangle \cap \Pi_{\mu \in M}\left\langle y_{\mu}\right\rangle\right) \oplus\langle c\rangle$. However, the commutative diagram

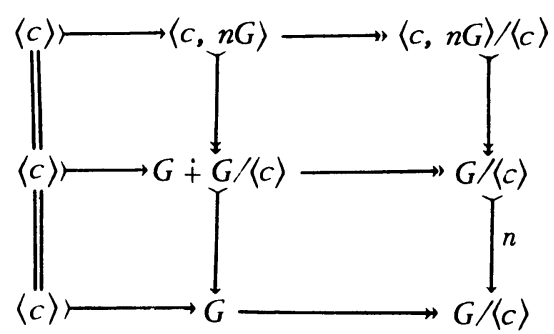

shows that $(* *)$ is the $n$-fold of $(*)$; the map $\langle c, n G\rangle \rightarrow G+G /\langle c\rangle$ is defined by $m c+n g \rightarrow(m c+n g, g+\langle c\rangle)$ for any integer $m$. The middle row represents the pullback construction. Since $(* *)$ splits, it represents the zero element of $\operatorname{Ext}(P, Z)$. Thus $(*)$ represents an element of $\operatorname{Ext}(P, Z)$ having finite order 
(a factor of $n$ ), which is a contradiction on the choice of $(*)$. The theorem is proved, and we point out that the example $G$ constructed admits a generalized discrete order for which the convex subgroups have order type $\omega+1$.

4. Some properties of generalized discrete groups. The lexicographic product and the lexicographic sum of copies of the integers are obviously g.d. groups. Furthermore, any free abelian group $F$ can be ordered so that $F$ is a lexicographic sum of copies of $Z$. From this it follows that if $G$ is any g.d. group and if $F^{\prime}$ is a free abelian group with $F$ a subgroup such that $F^{\prime} / F \simeq G$, then $F$ can be ordered as above and $F^{\prime}$ can be ordered so that its skeleton is completely determined by the skeletons of $F$ and $G$, respectively. Thus any g.d. group is the order homomorphic image of a g.d. group that is algebraically free.

We observe next, that if $G$ is a totally ordered abelian group and if $H$ is a subgroup of $G$, then the skeletons of $G$ and $H$ are closely related. For if $x$ is a nonzero element of $H$, consider the jump in $H$ and in $G$ determined by $x$. Denote by $U_{H}(x)$ and $L_{H}(x)$, respectively, the smallest convex subgroup of $H$ containing $x$, and the largest convex subgroup of $H$ not containing $x$. Let $U(x)$ and $L(x)$ be the corresponding convex subgroups of $G$. We want to observe that $U_{H}(x) / L_{H}(x)$ is order isomorphic to a subgroup of $U(x) / L(x)$. If $L_{H}^{\prime}(x)=L(x) \cap$ $H$ and $U_{H}^{\prime}(x)=U(x) \cap H$, then, trivially, $L_{H}^{\prime}(x)$ and $U_{H}^{\prime}(x)$ are convex subgroups of $H$ such that $x \notin L_{H}^{\prime}(x)$ and $x \in U_{H}^{\prime}(x)$. Thus we have

$$
L_{H}^{\prime}(x) \subseteq L_{H}(x) \subseteq U_{H}(x) \subseteq U_{H}^{\prime}(x) .
$$

Clearly $U_{H}^{\prime}(x) / L_{H}^{\prime}(x)$ is a subgroup of $U(x) / L(x)$. Since $U(x) / L(x)$ is archimedean, it follows that $U_{H}^{\prime}(x) / L_{H}^{\prime}(x)$ is archimedean, and therefore $U_{H}^{\prime}(x)=U_{H}(x)$ and $L_{H}^{\prime}(x)=L_{H}(x)$. In particular, if $U(x) / L(x) \simeq Z$, it follows that $U_{H}(x) / L_{H}(x)$ $\simeq Z$ and the following proposition is immediate.

Proposition 4.1. A subgroup of a generalized discrete group is again a generalized discrete group.

Remark. Proposition 4.1 implies that for any exact sequence $0 \rightarrow A \stackrel{a}{\longrightarrow}$ $G \rightarrow B \stackrel{\beta}{\longrightarrow} 0$, where $A, G$ and $B$ are ordered abelian groups and $\alpha$ and $\beta$ are $o$-homomorphisms, then $G$ is a g.d. group if and only if $A$ and $B$ are g.d. groups.

A totally ordered abelian group of finite torsion free rank is also of finite order rank [25, p. 50]. In [25, p. 49] it is observed that a g.d. group of finite order rank is a lexicographic sum of finitely many copies of $Z$. Thus if $H$ is of finite torsion free rank and if $H$ is a subgroup of a generalized discrete group, then in particular, $H$ is free. Applying Pontryagin's theorem [11, p. 51], we have the following corollary to Proposition 4.1 . 
Corollary 4.2. If $G$ is a generalized discrete group, then any countable subgroup of $G$ is algebraically free.

In general, if $G$ is a totally ordered abelian group such that $G=A \oplus B$, where $B$ is a convex subgroup of $G$, then an element $g=a+b$, where $a \in A$ and $b \in B$, is positive if and only if $a>0$ when $a \neq 0$ or $b>0$ when $a=0$. In other words, if $G=A \oplus B$ splits algebraically where the summand $B$ is a convex subgroup then this decomposition of $G$ is such that $G$ is the lexicographic sum of $A$ and $B$. This observation leads to the following corollary of Proposition 4.1 .

Corollary 4.3. If $G$ is a generalized discrete group and $H$ is a convex subgroup of $G$ such that $G / H$ is countable, then $G$ is the lexicograpbic sum $G=$ $K \oplus H$, where $K$ is some subgroup of $G$. In particular, if $G$ is countable, then any convex subgroup is a lexicograpbic summand of $G$.

Proof. The proof follows immediately by applying the well-known result that if $G / H$ is free then $G$ decomposes as $G=K \oplus H$ for some subgroup $K$ of $G$.

If $n$ is a positive integer and $G$ is a totally ordered abelian group, then clearly $G$ and $n G$ are order isomorphic. Using this fact and Proposition 4.1 we obtain the following trivial corollary, which will be needed in the next section.

Corollary 4.4. If $G$ is a totally ordered abelian group and if $H$ is a generalized discrete subgroup of $G$ containing $n G$ for some positive integer $n$, then $G$ is a generalized discrete group.

The following is an immediate consequence of the preceding results and a recent the orem of Hill [16].

Corollary 4.5. If $G$ is a generalized discrete group with countable skeleton, and if each principal convex subgroup of $G$ is algebraically free, then $G$ is algebraically free.

We now construct two examples of g.d. groups. There are two opposite situations we would like to illustrate.

Example 1. Let $G_{1}$ be the direct sum of copies of the integers $Z_{a}$ where $a \in L$ and $L$ is the positive integers ordered under the usual ordering. Order $G_{1}$ lexicographically. Then the convex subgroups of $G$ form a descending sequence $H_{0} \supset H_{1} \supset H_{2} \supset \ldots \supset H_{n} \supseteq \ldots$ where $H_{n}=\Sigma_{n \leq a} Z_{a}$ for all $n \geq 0$. In particular, for each convex subgroup $H$ there is a convex subgroup $K$ immediately below $H$, that is, $H$ covers $K$. Note that there is no smallest convex subgroup properly containing 0 .

Example 2. Here let $L$ be the positive integers inversely ordered, and let 
$G_{2}=\Sigma_{\alpha \in L} Z_{\alpha}$ ordered lexicographically. Then, in this case, the convex subgroups of $G_{2}$ form an ascending chain $H_{0} \subset H_{1} \subset \ldots \subset H_{n} \subset \ldots$, where $H_{n}=$ $Z_{n} \oplus \ldots \oplus Z_{2} \oplus Z_{1}$ for all $n \geq 0$. In this example, given any convex subgroup $K$ there is another convex subgroup $H$ which covers $K$.

Example 2 motivates the consideration of totally ordered abelian groups whose set of convex subgroups is well ordered with respect to containment. In particular, the convex subgroups of such groups can be indexed by ordinal numbers where $K_{\alpha} \subseteq K_{\beta}$ if and only if $\alpha \leq \beta$. Furthermore, given any proper convex subgroup $K_{\alpha}$ there is a convex subgroup $K_{\alpha+1}$ which covers $K_{\alpha}$.

Proposition 4.5. If $G$ is a generalized discrete group such that the convex subgroups are well ordered with respect to containment, then $G$ is a lexicographic sum of copies of $Z$.

Proof. We use transfinite induction on the ordinal number associated with the set of convex subgroups of $G$. If $K_{\alpha}$ is a convex subgroup of $G$ properly contained in $G$, then there is a convex subgroup $K_{a_{+}}$that covers $K_{a}$. Also, $K_{\alpha+1}=C_{a} \oplus K_{\alpha}$, where $C_{\alpha} \simeq Z$ and the decomposition is a lexicographic sum. In the indexing of convex subgroups of $G$, if $G=K_{\beta}$ and $\beta-1$ exists then $G=$ $C_{\beta-1} \oplus K_{\beta-1}$ and the inductive hypothesis implies $G$ is a lexicographic sum of copies of $Z$. If $\beta$ is a limit ordinal, then we apply the standard argument to conclude that $G$ is the algebraic direct sum of $C_{\alpha}$ for $\alpha<\beta$. By the inductive hypothesis any proper convex subgroup $K_{\lambda}$ is the lexicographic sum of $C_{\alpha}$ for $a<\lambda$.

Any nonzero element $x$ of $G$ can be written as $x=x_{a_{k}}+\cdots+x_{a_{1}}$, where $x_{\alpha_{i}}$ is a nonzero element of $C_{a_{i}}$ for each $i$ and $\alpha_{1}<\alpha_{2}<\ldots<\alpha_{k}$. Thus, it follows that $x \in K_{\lambda}$ where $\lambda>\alpha_{k}$. Since $K_{\lambda}$ is the lexicographic sum of the $C_{a}^{\prime}$ s, $x$ is positive if and only if $x_{a_{k}}$ is positive in $C_{a_{k}}$. Thus $G$ is the lexicographic sum of the $C_{a}$ 's.

We define a totally ordered abelian group $G$ to be $w$-discrete if every order homomorphic image of $G$ has a least positive element.

If $G$ is a $w$-discrete totally ordered abelian group, then obviously the convex subgroups of $G$ are also well ordered with respect to containment. Thus we have the following result from Proposition 4.5.

Proposition 4.6. If $G$ is a w-discrete totally ordered abelian group, then $G$ is the lexicographic sum of copies of the integers, where the summands are indexed by an initial segment of ordinal numbers inversely ordered. Conversely, any sucb lexicographic sum is a w-discrete group.

Obviously, a $w$-discrete group is a g.d. group. Further, a totally ordered 
abelian group $G$ of finite order rank is $w$-discrete if and only if $G$ is a g.d. group. That a g.d. group need not be $w$-discrete is seen from Example 1 above.

Note that any subgroup of a $w$-discrete group is again $w$-discrete. Thus if $G$ is a lexicographic sum of copies of $Z$ indexed by a suitable choice of indexing set then any subgroup is also a lexicographic sum of copies of $Z$.

5. An embedding theorem for generalized discrete groups. We have seen in $\$ 3$ that there are regular groups and generalized discrete groups which cannot be embedded in the lexicographic product of their components. Nevertheless, this embedding is possible for countable g.d. groups.

Theorem 5.1. Any countable generalized discrete group can be embedded in the lexicograpbic sum of its components.

We shall need the following lemma for the proof.

Lemma. Let $G$ be any countable generalized discrete group and let $g$ be an element of $G$. Then there exists a countable extension $E$ of $G$ by a (bounded) torsion group such that $E$ has a lexicographic decomposition $E=E_{1} \oplus E_{2} \oplus E_{3}$ where $E_{2}=\langle x\rangle$ is cyclic and some multiple of the generator $x$ is the given element $g$. Either or both of $E_{1}$ and $E_{3}$ may be zero.

Proof. Let $U(g)=\langle c\rangle \oplus L(g)$, where $U(g)$ is the smallest convex subgroup of $G$ containing $g$ and where $L(g)$ is the largest convex subgroup of $G$ not containing $g$. Since $G$ is a countable generalized discrete group, every convex subgroup of $G$ is a direct summand of $G$ according to Corollary 4.3, so let $G=E_{1} \oplus U(g)$. Write $g=n c+b$, where $n$ is an integer and $b \in L(g)$; we may choose the generator $c$ such that the integer $n$ is positive. Now embed $L(g)$ into an isomorphic copy $E_{3}$ of itself such that $n E_{3}=L(g)$. Choose $g_{3} \in E_{3}$ such that $n g_{3}=b$ and set $x=c+g_{3}$. Define $E=E_{1} \oplus\langle x\rangle \oplus E_{3}$, and observe that this is a lexicographic decomposition.

Proof of Theorem 5.1. We shall use the above lemma repeatedly to obtain a sequence $G=G_{0} \subseteq G_{1} \subseteq \cdots \subseteq G_{n} \subseteq \cdots$ such that $G_{n+1}$ is a torsion extension of $G_{n}$ and such that $G^{*}=\bigcup_{n<\omega} G_{n}$ is a lexicographic sum of integers. First, we enumerate the elements of $G_{0}=G$; let

$$
G_{0}=\left\{g_{0,1}, g_{0,2}, \cdots, g_{0, n}, \cdots\right\} .
$$

By the lemma, there exists a $G_{1}$ that admits a lexicographic decomposition $G_{1}=$ $E_{1} \oplus E_{2} \oplus E_{3}$ where $E_{2}$ is cyclic and contains $g_{0,1}$. Now enumerate the elements of $G_{1}$; let

$$
G_{1}=\left\{g_{1,1}, g_{1,2}, \cdots, g_{1, n}, \cdots\right\}
$$


We can write $g_{1,1}=e_{1}+e_{2}+e_{3}$ where $e_{i} \in E_{i}$. Applying the lemma again to $E_{1}$ and $E_{3}$ using the elements $e_{1}$ and $e_{3}$, we have lexicographic decompositions $E_{1}=E_{11} \oplus E_{12} \oplus E_{13}$ and $E_{3}=E_{31} \oplus E_{32} \oplus E_{33}$, where $E_{12}$ and $E_{32}$ are cyclic containing $e_{1}$ and $e_{3}$, respectively. Observe that $g_{0,1}$ and $g_{1,1}$ are both contained in $E_{12} \oplus E_{2} \oplus E_{32}$. Now we proceed to the induction step. Suppose that we have a finite chain $G_{0} \subseteq G_{1} \subseteq \cdots \subseteq G_{n}$ of torsion extensions of $G_{0}$ such that for certain elements $g_{1}, g_{2}, \ldots, g_{n}$ belonging to $G_{n-1}$, we have a lexicographic decomposition

$$
G_{n}=\sum_{i \in I(n)} E_{n, i}
$$

indexed by some finite subset $I(n)$, such that $\left\langle g_{1}, g_{2}, \ldots, g_{n}\right\rangle \subseteq \Sigma_{j \in J(n)} E_{n, j}$ for some subset $J(n)$ of $I(n)$ with $E_{n, j}$ cyclic for each $j \in J(n)$. Now let $g_{n+1} \in G_{n}$ and write $g_{n+1}=\Sigma_{i \in I(n)} e_{i}$, where $e_{i} \in E_{n, i}$. For each $i \in I(n)-$ $J(n)$, let $F_{i}$ be a torsion extension of $E_{n, i}$ such that $F_{i}=F_{i, 1} \oplus\left\langle x_{i}\right\rangle \oplus F_{i, 3}$ where the sum is lexicographic and $e_{i} \epsilon\left\langle x_{i}\right\rangle$. If $j \in J(n)$, set $F_{j}=E_{n, j}$. Define $G_{n+1}=\Sigma_{i \in I(n)} F_{i}$, and observe that we have lexicographic sums

$$
G_{n+1}=\sum_{i \in I(n)} F_{i}=\sum_{i \in I(n+1)} E_{n+1, i},
$$

where the latter decomposition is the refinement of the first obtained from

$$
F_{i}=F_{i, 1} \oplus\left\langle x_{i}\right\rangle \oplus F_{i, 3} \cdot
$$

In view of our construction, there exists a subset $J(n+1)$ of $I(n+1)$ such that $E_{n+1, j}$ is cyclic if $j \in J(n+1)$ and such that $\left\langle g_{1}, g_{2}, \ldots, g_{n}, g_{n+1}\right\rangle \subseteq$ $\sum_{j \in J(n+1)} E_{n+1, j}$. Moreover, we can choose the index set such that $J(n) \subseteq$ $J(n+1)$ and such that $E_{n, j}=E_{n+1, j}$ if $j \in J(n)$.

Obviously, we can choose the sequence $\left\{g_{1}, g_{2}, \ldots, g_{n}, \ldots\right\}$ such that the set $\bigcup_{n<\omega} G_{n}$, a countable set, is exhausted. The proof is finished with the observation that the group $G^{*}=\bigcup_{n<\omega} G_{n}$ is a lexicographic sum of integers since it is equal to $\bigcup_{n<\omega}\left(\sum_{j \in J(n)} E_{n, j}\right)$; the latter is a lexicographic sum since $J(n) \subseteq J(n+1)$ and since $E_{n, j}=E_{n+1, j}$ if $j \in J(n)$ for each $n$.

6. Order separability. In this section we introduce the concept of an oseparable group. In the previous section we showed that a countable generalized discrete group is embeddable in the lexicographic sum of its components. In this section our main result is that such a group $G$ is, in fact, equal to the lexicographic sum of its components under the additional assumption that $G$ is order separable.

A totally ordered abelian group $G$ is said to be order separable (o-separable) if for every finite subset $S$ of $G$ there exists a lexicographic sum decomposition 
of $G, G=\Sigma_{i \in I} A_{i}$, where $I$ is a finite indexing set containing a subset $J$ such that $A_{j}$ is order isomorphic to a subgroup of $Q$ for each $j \in J$, and $S \subseteq \Sigma_{j \in J} A_{j}$. Clearly if $G$ decomposes as above, for $i_{0} \in I, \Sigma_{i_{0 \leq i}} A_{i}$ is a convex subgroup of $G$, and if $J$ is chosen to be minimal such that $S \subseteq \Sigma_{j \in J} A_{j}$ and if $j_{0}$ is the smallest element of $J$, then $\Sigma_{j_{0} \leq i} A_{i}$ is a principal convex subgroup of $G$ (determined by some element $s \in S$ ). In particular, note that any principal convex subgroup of an o-separable group $G$ is lexicographically a direct summand of $G$.

Observe that our definition of $G$ being o-separable generalizes the definition of Baer [2] of $G$ being separable for an unordered torsion free abelian group $G$.

Proposition 6.1. Let $G$ be an o-separable group and $H$ a convex subgroup of $G$. If $H$ is a direct summand of $G$, then $H$ is also o-separable.

Proof. Let $S$ be a finite subset of $H$ and suppose $G=\Sigma_{i \in I} A_{i}$ where $S \subseteq$ $\Sigma_{j \in J} A_{j}$ with $A_{j} \subseteq Q$ if $j \in J$. Let $J$ be minimal with respect to this property and let $j_{0}$ be the minimal index in $J$. Then $C=\Sigma_{j 0 \leq i} A_{i}$ is the minimal convex subgroup of $G$ containing $S$. Thus $C \subseteq H$ and $H=\left(\Sigma_{i \in I} A_{i}\right) \cap H=\left(H \cap \Sigma_{i<j_{0}} A_{i}\right)$ $\oplus C$, that is, $H=H_{1} \oplus \Sigma_{j 0 \leq i} A_{i}$ where $S \subseteq \Sigma_{j \in J} A_{j}$.

Proposition 6.2. The lexicographic sum of o-separable groups is o-separable.

Proof. Suppose $G=\Sigma_{\lambda \in \Lambda} H_{\lambda}$ where the order on $G$ is the lexicographic order and each $H_{\lambda}$ is o-separable. If $S$ is a finite subset of $G$, then for each $x \in S$ we project onto $H_{\lambda}$. Clearly there are only finitely many $\lambda$ 's, say $\left\{\lambda_{1}\right.$, $\left.\ldots, \lambda_{k}\right\}$, such that the projection of $x, \pi_{\lambda}(x)$, is nonzero in $H_{\lambda}$. Furthermore, assume $\lambda_{1}<\lambda_{2}<\cdots<\lambda_{k}$. For $\lambda_{t} \in\left\{\lambda_{1}, \ldots, \lambda_{k}\right\}$, let $S_{t}=\left\{x_{\lambda_{t}} \in H_{\lambda_{t}} \mid\right.$ $x_{\lambda_{t}} \neq 0$ and $x_{\lambda_{t}}=\pi_{\lambda_{t}}(x)$ for some $\left.x \in S\right\}$. Using the fact that each $H_{\lambda_{t}}$ is $o^{-}$ separable and that $S_{t}$ is a finite set, we decompose $H_{\lambda_{t}}, H_{\lambda_{t}}=\Sigma_{i \in I_{t}} A_{i}$ where $S_{t} \subseteq \Sigma_{j \in J_{t}} A_{j}$ and $A_{j}$ is a subgroup of $Q$ for $j \in J_{t} \subseteq I_{t}$. Then $H$ can be decomposed as

$\left(\sum_{\lambda<\lambda_{1}} H_{\lambda}\right) \oplus\left(\sum_{i \in I_{1}} A_{i}\right) \oplus\left(\sum_{\lambda_{1}<\lambda_{<}<\lambda_{2}} H_{\lambda}\right) \oplus \cdots \oplus\left(\sum_{i \in I_{k}} A_{i}\right) \oplus\left(\sum_{\lambda_{k}<\lambda} H_{\lambda}\right)$.

If $J=\bigcup_{t=1}^{k} J_{t}$, then clearly $S \subseteq \Sigma_{j \in J} A_{j}$.

We do not know if the lexicographic product of $o$-separable groups is again $o$-separable.

We are now ready to prove the main result of this section.

Theorem 6.3. If $G$ is a countable o-separable generalized discrete group, then $G$ is the lexicographic sum of its components.

Proof. Let $G=\left\{g_{1}, g_{2}, \ldots, g_{n}, \ldots\right\}$. For each positive integer $n$, let 
$S_{n}$ be a finite subset of $G$ containing $g_{1}, g_{2}, \ldots, g_{n}$. There exists a lexicographic decomposition $G=\Sigma_{i \in I(n)} H_{i}^{(n)}$, where $I(n)$ is a finite set, with the following properties: for some subset $J(n)$ of $I(n)$,

$$
S_{n} \subseteq \sum_{j \in J(n)} H_{j}^{(n)} \text { and } H_{j}^{(n)}=\left\langle b_{j}^{(n)}\right\rangle \text { for each } j \in J(n) .
$$

We shall always assume that $J(n)$ is minimal with respect to having the above properties. Moreover, we shall assume that the finite subsets $S_{n}$ of $G$ are chosen inductively in such a way that $b_{j}^{(n)} \in S_{n+1}$ for each $j \in J(n)$.

Now, we proceed to show that such decompositions exist with $H_{j}^{(n)}=H_{j^{\prime}}^{(n+1)}$ for each $j \in J(n)$; $j^{\prime}$ represents some element of $J(n+1)$. This is done by induction on $n$. Suppose that $H_{j}^{(k)}=H_{j^{\prime}}^{(k+1)}$ for $j \in J(k)$ if $k<n$.

Let $l$ be the largest element of $J(n)$, and write $b_{l}^{(n)}=\sum_{j \in J(n+1)} t_{j} b_{j}^{(n+1)}$, where $t_{j}$ is an integer. Let $s$ be the smallest element of $J(n+1)$ such that $t_{s}$ $\neq 0$. Then $b_{l}^{(n)}=\Sigma_{s \leq j} \epsilon_{J(n+1)} t_{j} b_{j}^{(n+1)}$. Note that

$$
\sum_{l \leq i \in I(n)} H_{i}^{(n)}=\sum_{s \leq i \in I(n+1)} H_{i}^{(n+1)}
$$

because each is the smallest convex subgroup of $G$ containing $b_{l}^{(n)}$. Moreover, $H_{l}^{(n)} \cong Z \cong H_{s}^{(n+1)}$, so

$$
\sum_{l<i \in I(n)} H_{i}^{(n)}=\sum_{s<i \in I(n+1)} H_{i}^{(n+1)}
$$

because each is the largest convex subgroup of $G$ not containing $b_{l}^{(n)}$.

Obviously (1) and (2) imply that

$$
\begin{aligned}
\sum_{s \leq i \in I(n+1)} H_{i}^{(n+1)} & =H_{l}^{(n)} \oplus \sum_{s<i \in I(n+1)} H_{i}^{(n+1)}, \\
\sum_{s \leq j \in J(n+1)} H_{j}^{(n+1)} & =H_{l}^{(n)} \oplus \sum_{s<j \in J(n+1)} H_{j}^{(n+1)} .
\end{aligned}
$$

Now, let $l(1)=l, s(1)=s$ and let $l(2)$ be the largest element of $J(n)$ less than $l(1)$. Write

$$
b_{l(2)}^{(n)}=\sum_{j \in J(n+1)} t_{j} b_{j}^{(n+1)}, \text { where } t_{j} \text { is an integer. }
$$

Let $s(2)$ be the smallest element of $J(n+1)$ such that $t_{s(2)} \neq 0$. Then $s(2)<$ $s(1)$ since $l(2)<l(1)$. From $b_{l(2)}^{(n)}=\Sigma_{s(2) \leq j} \epsilon J(n+1) t_{j} b_{j}^{(n+1)}$, we have

$$
\begin{aligned}
\sum_{l(2) \leq i \in I(n)} H_{i}^{(n)} & =\sum_{s(2) \leq i \in I(n+1)} H_{i}^{(n+1)}, \\
\sum_{l(2)<i \in I(n)} H_{i}^{(n)} & =\sum_{s(2)<i \in I(n+1)} H_{i}^{(n+1)}
\end{aligned}
$$


the latter equality holds because $H_{l(2)}^{(n)} \cong Z \cong H_{s(2)}^{(n+1)}$. Now, we have

$$
\begin{aligned}
& \sum_{s(2) \leq i \in I(n+1)} H_{i}^{(n+1)}=H_{l(2)}^{(n)} \oplus \sum_{s(2)<i \in I(n+1)} H_{i}^{(n+1)}, \\
& \sum_{s(2) \leq j \in J(n+1)} H_{j}^{(n+1)}=H_{l(2)}^{(n)} \oplus \sum_{s(2)<j \in J(n+1)} H_{j}^{(n+1)} \\
& =H_{l(2)}^{(n)} \oplus \sum_{s(2)<j<s(1) ; j \in J(n+1)} H_{j}^{(n+1)} \oplus H_{l(1)}^{(n)} \oplus \sum_{s(1)<j \in J(n+1)} H_{j}^{(n+1)} .
\end{aligned}
$$

Continuing in this way we show we can choose the decomposition $\Sigma_{j \in J(n+1)} H_{j}^{(n+1)}$ such that $H_{j}^{(n)}=H_{j^{\prime}}^{(n+1)}$ for each $j \in J(n)$. Therefore, we conclude that $G=$ $\Sigma\left\langle b_{j}^{(k)}\right\rangle$. Furthermore, if we rename the $b_{j}^{(k)}$ as $c_{\alpha}$ where $\alpha<\beta$ if $c_{\alpha}>c_{\beta}$, then $G=\Sigma\left\langle c_{\alpha}\right\rangle$. It follows that this is a lexicographic sum. For suppose $g_{n}$ $\in G$ and $x_{a} \in\left\langle c_{a}\right\rangle$ is the first nonzero coordinate of $g_{n}$. Now $g_{n} \in S_{n} \subseteq$ $\Sigma_{j \in J(n)}\left\langle b_{j}^{(n)}\right\rangle$ and $c_{a}=b_{j}^{(n)}$ for some $j \in J(n)$. This last decomposition is a lexicographic sum. This fact implies that $g_{n}$ is positive if and only if $x_{\alpha}$ is positive.

7. A useful example. We present now an example of a subgroup $H$ of the lexicographic sum of countably many copies of $Z$ such that $H$ is not a lexicographic sum of copies of $Z$. This example also shows that o-separability is not a hereditary property. Another use of the example is to show that the conclusion of our embedding theorem (Theorem 5.1) cannot be strengthened to conclude that a countable g.d. group is a lexicographic sum of copies of $Z$.

Let $G=\Sigma_{1 \leq i<\omega}\left\langle x_{i}\right\rangle$ where $Z \simeq\left\langle x_{i}\right\rangle$ and $G$ is ordered lexicographically. Let $H=2 x_{1} \oplus \Sigma_{1 \leq i<\omega}\left\langle x_{i}+x_{i+1}\right\rangle$ with the induced order on $H$. Clearly $H$ is a subgroup of $G$ of index 2. Thus there is a one-to-one correspondence between convex subgroups of $G$ and convex subgroups of $H$. If $C$ is a convex subgroup of $G$, then $C \cap H$ is a convex subgroup of $H$. Furthermore, if $C^{\prime}$ is a convex subgroup of $H$, then $C=\left\{x \in G \mid 2 x \in C^{\prime}\right\}$ is the convex subgroup of $G$ such that $C \cap H=C^{\prime}$.

Suppose that $H$ is the lexicographic sum $\Sigma_{1 \leq i<\omega}\left\langle y_{i}\right\rangle$ where each $y_{i}$ is positive. Under the above correspondence of convex subgroups of $G$ and $H$, we see that the chain of convex subgroups of $H$ is

$$
H=U_{H}\left(2 x_{1}\right) \supset U_{H}\left(2 x_{2}\right) \supset \cdots \supset U_{H}\left(2 x_{i}\right) \supset \ldots
$$

Thus we may assume that $y_{i}>y_{j}$ for $i<j$, in the lexicographic sum $H=$ $\Sigma_{1 \leq i<\omega}\left\langle y_{i}\right\rangle$, and that $U_{G}\left(y_{i}\right)=U_{G}\left(x_{i}\right)$.

If $y_{1}=t_{11} x_{1}+\cdots+t_{1 k_{1}} x_{k_{1}}$, we see that $t_{11} \neq 0$ since $U\left(y_{1}\right)=U\left(x_{1}\right)$. Similarly, $y_{2}=t_{21} x_{1}+t_{22} x_{2}+\cdots+t_{2 k_{2}} x_{k_{2}}$ and we conclude $t_{21}=0$ and $t_{22}$ $\neq 0$. Continuing, we have 


$$
\begin{array}{lc}
y_{1}=t_{11} x_{1}+t_{12} x_{2}+\cdots+t_{1 k_{1}}{ }^{x} k_{1} \\
y_{2}= & t_{22}{ }^{x_{2}}+\cdots+t_{2 k_{2}}{ }^{x_{k_{2}}} \\
\vdots & \\
y_{m}= & t_{m m}{ }^{x}{ }_{m}+\cdots+t_{m k_{m}}{ }^{x} k_{m}
\end{array}
$$

where $t_{i i} \neq 0$ for each $i$. In fact, $t_{i i}>0$ if $x_{i}$ is chosen positive.

We observe $t_{11}=1$. For

$$
x_{1}+x_{2}=s_{1} y_{1}+\cdots+s_{k} y_{k}=s_{1} t_{11} x_{1}+\left(s_{2} t_{22}+s_{1} t_{12}\right) x_{2}+\cdots
$$

and, by the uniqueness of representation, $s_{1} t_{11}=1$. In the representation $x_{2}+$ $x_{3}=s_{1} y_{1}+s_{2} y_{2}+\cdots+s_{l} y_{l}$ we see that $s_{1}=0$ since, otherwise, $U\left(x_{2}+x_{3}\right)$ $=U\left(x_{2}\right)=U\left(y_{1}\right)$. Thus

$$
x_{2}+x_{3}=s_{2} y_{2}+\cdots=s_{2} t_{22} x_{2}+\text { terms involving } x_{j} \text { for } j>2 .
$$

From this it follows $t_{22}=1$. By induction we see that $t_{i i}=1$ for each $i$. However, $2 x_{1} \in H$ and

$$
\begin{array}{r}
2 x_{1}=s_{1} y_{1}+\cdots+s_{m} y_{m}=s_{1} t_{11} x_{1}+\left(s_{2} t_{22}+s_{1} t_{12}\right) x_{2} \\
+\cdots+\left(\sum_{i=1}^{k} s_{i} t_{i k}\right) x_{k}+\cdots+\sum_{i=1}^{m} s_{i} t_{i l} x_{l}+\cdots
\end{array}
$$

where $k<m, l \geq m$. But $t_{11}=1$ implies $s_{1}=2$. Then $s_{2} t_{22}+s_{1} t_{12}=0$ and $t_{22}=1$ imply $s_{2}$ is even. Assuming $s_{k-1}$ is even for $2<k \leq m$, then $\sum_{i=1}^{k} s_{i} t_{i k}=0$ and $t_{k k}=1$ imply $s_{k}$ is even. Hence $s_{1}, \ldots, s_{m}$ are all even, and $2 x_{1}=2\left(y_{1}+s_{1}^{\prime} y_{2}+\cdots+s_{m}^{\prime} y_{m}\right)$. But this implies $x_{1} \in H$. This contradiction shows $H$ is not a lexicographic sum.

Proposition 6.2 implies that $G$ is o-separable. But because of Theorem 6.3, $H$ is not o-separable.

8. Application of generalized discrete groups to valuation rings. Butts and Gilmer [4] defined a valuation ring $V$ to be discrete if the only primary ideals of $V$ are prime powers, and they proved: A valuation ring is discrete if and only if its value group is generalized discrete. Using the result of $\$ 4$, we can obtain easy group theoretic proofs of the following two results, which were proved by R. Gilmer in [12] using ideal theory.

Corollary 8.1. Let $V$ be a valuation ring with quotient field $K, L$ is a finite field extension of $K$, and $V^{\prime}$ a valuation ring of $L$ sucb that $V^{\prime} \cap K=V$. Then if $V$ is a discrete valuation ring so is $V^{\prime}$.

Corollary 8.2. Let $V$ be a valuation ring of $K$ and $F$ a subfield of $K$. If $V$ is a discrete valuation ring of $K$, then $V \cap F$ is a discrete valuation ring of $F$. 


\section{REFERENCES}

1. S. Abhyankar, Ramification theoretic methods in algebraic geometry, Ann. of Math. Studies, no. 43, Princeton Univ. Press, Princeton, N. J., 1959. MR 21 \#4158. $68-122$.

2. R. Baer, Abelian groups without elements of finite order, Duke Math. J. 3 (1937),

3. B. Banaschewski, Totalgeordnete Moduln, Arch. Mat. 7 (1957), 430-440.

MR 19, 385.

4. H. S. Butts and R. W. Gilmer, Jr., Primary ideals and prime power ideals, Canad.

J. Math. 18 (1966), 1183-1195. MR 37 \#204.

5. A. H. Clifford, Note on Hahn's theorem on ordered abelian groups, Proc. Amer.

Math. Soc. 5 (1954), 860-863. MR 16, 792.

6. P. F. Conrad, Embedding theorems for abelian groups with valuations, Amer. J. Math. 75 (1953), 1-29. MR 14, 842.

7. - A note on valued linear spaces, Proc. Amer. Math. Soc. 9 (1958), 646-647. MR $20 \# 5808$.

8. - Representations of partially ordered abelian groups as groups of real valued functions, Acta Math. 116 (1966), 199-221. MR 34 \#1418.

9. P. F. Conrad, J. Harvey and C. Holland, The Hahn embedding theorem for abelian lattice-ordered groups, Trans. Amer. Math. Soc. 108 (1963), 143-169. MR 27 \#1519.

10. L. Fuchs, Partially ordered algebraic systems, Pergamon Press, Oxford; Addison-Wesley, Reading, Mass., $1963 . \quad$ MR 30 \#2090.

11. - A belian groups, Internat. Series of Monographs on Pure and Appl. Math., Pergamon Press, New York, 1960. MR 22 \#2644.

12. R. W. Gilmer, Jr., Multiplicative ideal theory, Queen's Papers in Pure and Appl. Math., no. 12, Queen's University, Kingston, Ont., 1968. MR 37 \#5198.

13. K. A. H. Gravett, Ordered abelian groups, Quart. J. Math. Oxford Ser. (2) 7 (1956), 57-63. MR 19, 1037.

14. H. Hahn, Über die nichtarchimedischen Grossensysteme, S.•B. Akad. Wiss. Wien. IIa 116 (1907), 601-655.

15. M. Hausner and J. G. Wendel, Ordered vector spaces, Proc. Amer. Math. Soc. 3 (1952), 977-982. MR 14, 566.

16. P. Hill, On the freeness of abelian groups: a generalization of Pontryagin's theorem, Bull. Amer. Math. Soc. 76 (1970), 1118-1120. MR 41 \#8518.

17. K. Iwasawa, On linearly ordered groups, J. Math. Soc. Japan 1 (1948), 1-9. MR 10, 428.

18. F. Loonstra, Discrete groups, Nederl. Akad. Wetensch. Proc. Ser. A $54=$ Indag. Math. 13 (1951), 162-168. MR 13, 13.

19. R. J. Nunke, Slender groups, Acta Sci. Math. (Szeged) 23 (1962), 67-73. MR $26 \# 2508$.

20. P. Ribenboim, Sur les groupes totalement ordonnés et l'arithmétique des anneaux de valuation, Summa Brasil. Math. 4 (1958), 1-64. MR 21 \#6396.

21. - Théorie des groupes ordonnés, University Press, Bahia Blanca, Argentina, 1959.

22. N. Sankaran, Classification of totally ordered abelian groups, J. Indian Math. Soc. 29 (1965), 9-29. MR 32 \#5748.

23. N. Sankaran and R. Venkataraman, A generalization of the ordered group of integers, Math. Z. 79 (1962), 21-31. MR 25 \#1224.

24. A. Robinson and E. Zakon, Elementary properties of ordered abelian groups, Trans. Amer. Math. Soc. 96 (1960), 222-236. MR 22 \#5673.

25. O. Zariski and P. Samuel, Commutative algebra. Vol. 2, University Series in Higher Math., Van Nostrand, Princeton, N. J., 1960. MR 22 \#11006. 32306

DEPARTMENT OF MATHEMATICS, FLORIDA STATE UNIVERSITY, TALLAHASSEE, FLORIDA 